\title{
Feasibility of Using Physiological Signals from a Wearable Biosensor to Monitor Dehydration of Construction Workers
}

\author{
Amit Ojha ${ }^{1}$, Shahrad Shakerian ${ }^{2}$, Mahmoud Habibnezhad ${ }^{3}$, Houtan Jebelli ${ }^{4}$, \\ SangHyun Lee ${ }^{5}$ and Mohammad Sadra Fardhosseini ${ }^{6}$
}
1 Pennsylvania State University, University Park, PA, USA, azo5242@psu.edu
2 Pennsylvania State University, University Park, PA, USA, sqs6689@psu.edu
3 Pennsylvania State University, University Park, PA, USA, mjh6946@psu.edu
4 Pennsylvania State University, University Park, PA, USA, hkj5117@psu.edu
5 University of Michigan, Ann Arbor, MI, USA, shdpm@umich.edu
6 University of Washington, Seattle,WA, USA, sadrafh@uw.edu

\begin{abstract}
Among major industries, due to the labor-intensive nature of most of the construction tasks, the construction industry has some of the highest numbers of heat-related illness claims. The leading cause of these illnesses is the repeated exposure of workers to heat stress, which adversely affects productivity, safety, and health. A biophysical interpretation of the body's responses to heat stress is a promising way to continuously measure the likelihood of heat stress exposure of workers. Such a method surpasses current metrics such as Wet Bulb Globe Temperature and Heat Index. The others do not account for variations of individual physiology and biometrics (e.g., age, gender, and metabolism) in response to heat stress. Also, environmentally based methods cannot be used to continuously monitor heat stress in the workplace. This study aims to examine the effect of heat stress exposure on the flux of three different physiological signals: photoplethysmography (PPG), electrodermal activity (EDA), and skin temperature (S.T). To facilitate capture of workers' physical responses to acute heat, these are acquired from a wristband biosensor. To that end, physiological data were gathered from 10 workers performing construction tasks under three climatic conditions, each with a different likelihood of exposure to heat stress - conditions of caution, extreme caution, and danger, as defined by the National Oceanic and Atmospheric Administration (NOAA)'s National Weather Service. Heart rate, heart rate variability, electrodermal activity, electrodermal response, and mean skin temperature were extracted to examine the potential of these signals for measuring workers' heat stress. The results indicated statistically significant differences in the metrics of heat stress exposure. The findings demonstrated the feasibility of PPG, EDA, and S.T. in capturing physiological changes during heat stress exposure and dehydration.
\end{abstract}

(c) 2020 The Authors. Published by Budapest University of Technology and Economics \& Diamond Congress Ltd Peer-review under responsibility of the Scientific Committee of the Creative Construction Conference 2020.

Keywords: dehydration assessment, wearable biosensors, physiological monitoring

\section{Introduction}

The construction industry is beleaguered by extensive health and safety issues. At labor-intensive construction sites, workers perform physically arduous tasks under hazardous working conditions all day [1-3]. Frequently done in scorching and unmonitored environments without enough of a break to recover physiologically from overexertion, tasks are often performed with limited or lack of shade or water [4], and workers can suffer dehydration, as well as heat stress. 
In fact, enough exposure to heat can cause acute illnesses, such as dehydration, and chronic diseases, such as brain damage, both of which can adversely affect workers' productivity, safety, and health [5-8]. Between 2000 and 2010, construction was responsible for $36.8 \%$ of heat-related deaths, highest in the United States [9], a risk of heat-related fatalities 13 times greater than other industries [10]. Therefore, monitoring worker's heat stress exposure should be diligently implemented in health and safety management at construction sites.

To prevent heat-related illnesses, systematic heat stress measurement is of the utmost importance. There have been several efforts to gauge such. Some use answers to questionnaires [11,12]. However, surveybased methods interfere with ongoing tasks and are prone to biases. Other heat stress measurements rely on environmentally based metrics, such as Wet Bulb Globe Temperature (WBGT) and Heat Index (H.I.) $[11,13]$. Despite their use in measuring the state of heat stress in the field, these metrics cannot assess the full variation in a worker's physical characteristics and personal physiology $[14,15]$. Therefore, there is a need for objective and non-intrusive ways to evaluate heat stress in the field.

Biophysical responses to a workplace stimulus can offer valuable information about a worker's health, mental state, and other work-related responses [16-19]. Recent advances in wearable technologies offer an unprecedented opportunity to do non-intrusive, objective, and continuous real-time monitoring of workers' physical activity and processes [17,18,20,21]. These aspects of physiology can be monitored easily using photoplethysmography (PPG), electrodermal activity (EDA), and skin temperature (S.T.). The responses can be promptly processed and effectively analyzed to infer health conditions $[16,22,23]$. Specifically, this analysis can pave the way for continuous heat stress assessment in the field. In this vein, this study attempts to investigate various features of PPG, EDA, and S.T. signals as mechanisms of heat stress exposure.

\section{Current methods of heat stress assessment and their limitations}

Construction practitioners and researchers have mainly relied on subjective techniques (survey-based methods) to assess heat stress at work sites. The Heat Strain Score Index (HSSI) is a widely used subjective technique for heat stress assessment. In this method, heat strain experienced by an individual is evaluated by a questionnaire that contains 17 items related to the assessment of heat stress, such as questions about the experience of thermal and humidity conditions, the intensity of sweating, and intensity of fatigue [11]. While this method has broadened our understanding of the heat-stress of construction workers, it is prone to biases of self-report inherent in subjective scales. Also, doing the survey will interrupt the workflow because workers are required to participate during work hours. In addition to subjective assessment, heat stress has also been understood in terms of meteorological (climatic) parameters. Metrics such as H.I. and WBGT can monitor heat stress at construction sites. By assessing two common meteorological values, temperature, and relative humidity, H.I. reveals the climatic condition as experienced by the human body [13]. This index ignores other meteorological parameters (i.e., wind speed, air pressure, and sunlight) to evaluate the climatic conditions affecting heat stress accurately. WBGT is an efficient assessment of the thermal environment (ambient temperature, relative humidity, wind, and solar radiation) [13]. It is the most widely used index in assessing heat stress and has been adopted by the U.S. occupational safety and health authorities and other national and international agencies as the basis of heat-stress standards [24]. Although WBGT is a convenient method, the chances of heat stress exposure being different from environmental conditions and the reliability of the worker's judgment of his/her metabolic rate reduces its viability. More importantly, along with other environmentally-based techniques, WBGT suffers from generalization, representing only one value for heat stress exposure. Workers' responses to such exposure are different due to variations in physiological status such as age, gender, chronic or pre-existing diseases, fitness, hydration, and use of alcohol and medication. Different types of physical activity, body size acclimation, and clothing vary across workers [24-28], pointing to the importance of physiologically based techniques as a solution to heat-stress assessment. 


\section{Knowledge gaps and research objectives}

As mentioned above, current techniques mainly rely on subjective evaluation, and environmental assessment for measuring heat stress exposure, a more in-depth evaluation of workers' physiological conditions in the field is necessary for efficient and pragmatic monitoring. With current advances in nonintrusive wearable biosensors, several physiological responses to heat-related stimuli can be captured by a physiological sensor. These biosignals contain valuable information regarding physiological functioning and changes, and they can be extracted with advanced signal processing and machine learning techniques. However, the extent to which the biosignals can reveal the correct fluxes in human physiology depends on the type of stimulus.

Previous studies suggested PPG, EDA, and S.T. could capture information about the body's thermoregulation and be used as physiological signals [29-31]. PPG is a noninvasive way to measure blood volume changes in microscale vessels just under the skin [23]. When individuals are in a highly stressful environment, the brain innervates the heart via the sympathetic nervous system. Changes in the heartbeat (H.B.) mechanism will result in variations in blood volume; these can be measured by PPG [32]. Metrics obtained from PPG signals (e.g., H.B. variation) have the potential to measure workers' exposure to heatrelated injuries (heat stroke and exhaustion) [33,34]. As a result, PPG can provide an opportunity for understanding workers' bodily responses to thermal stress.

The sympathetic nervous system controls the sweat glands. EDA measures the changes in the electrical properties of the skin in response to autonomic sweat gland activity [35]. If the sympathetic branch of the autonomic nervous system is activated by external stressors, the number of active sweat glands increases, reflecting a higher EDA measurement [36], several features can be extracted from it, such as mean value, median value, variance, electrodermal level [EDL], and electrodermal response [EDR]) to measure these changes. To this end, EDA can be introduced as a reliable signal of the sympathetic nervous system when thermally stressful environments produce dehydration. When the body is subjected to intense, acute thermal conditions, the thermoregulation system adjusts skin temperatures through vasodilation and constriction to maintain thermal homeostasis [37-39]. The dynamic between these two mechanisms controls skin blood flow, resulting in skin temperature adjustments. S.T. has the potential to be a quantitative and objective indicator of likely illness and injuries $[33,39,40]$. Features like mean skin temperature (MST), mean frequency, and median frequency can be extracted from S.T. to assess the heat shock experienced by workers during ongoing work.

As mentioned above, the stimulation of the sympathetic autonomic nervous system results in significant changes in EDA, S.T., and PPG [16]. Nevertheless, the feasibility of using these measures to understand workers' heat stress under various conditions has not been well studied. Therefore, this paper aims to investigate more precisely the potential of these biosignals in unfolding the responses of construction workers to levels of heat stress exposure.

To this end, the authors recruited ten healthy subjects to perform two physical activities under three climatic conditions, namely low, medium, and high exposure to heat stress. During the experimental procedures, the subjects' physiological signals were collected from wearable sensors. A wide variety of metrics were extracted from the signals, such as heart rate (H.R.), heart rate variability (HRV), EDL, EDR, and mean skin temperature (MST), to name a few.

By statistically analyzing these features, a more in-depth understanding of the potential responses of a worker's body to heat stress can be achieved. Furthermore, such comparisons can reveal the underlying effect of heat stress on various aspects of PPG, EDA, and S.T. biosignals. Also, future studies can exploit the findings of this one to more suitably select relevant biosignals to develop predictive models.

\section{Experimental design}

\subsection{Subjects}

Experimental procedures were established to collect physiological signals from 10 able-bodied adults recruited among students at Pennsylvania State University (PSU). The data collection protocol was approved by the Institutional Review Board (IRB) at PSU. All subjects were provided with informed consent forms 
explaining the confidentiality of the data and participants' rights. After the consent form was signed and received, all subjects were asked to provide demographic information (i.e., age, gender, height, and weight). To exclude unhealthy subjects, participants were also asked for any history of health problems. None of the subjects reported any. Among ten subjects, seven were male; the remaining three were female. No subjects had any clinical conditions.
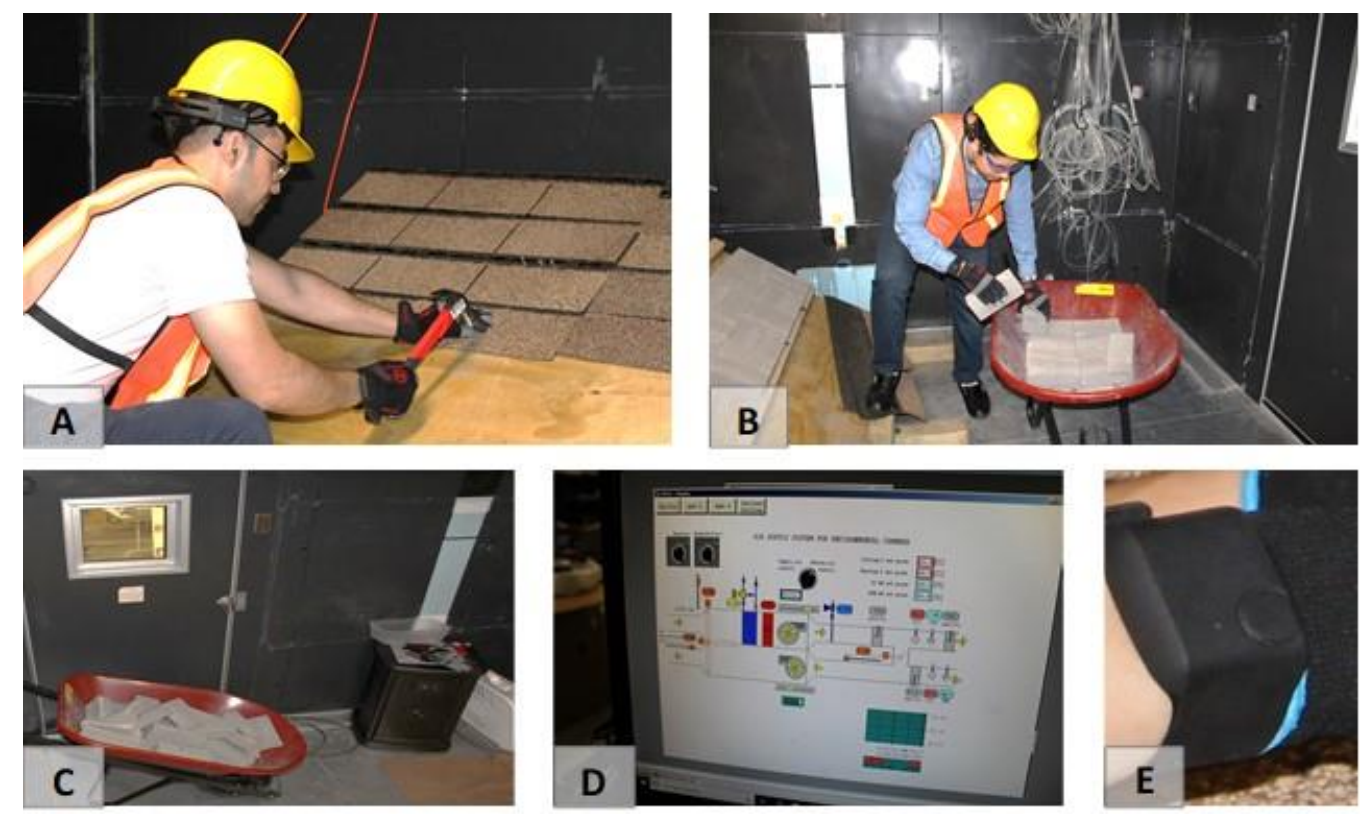

Fig. 1. Experimental setup: (a) roofing task; (b) materials handling task; (c) chamber room; (d) control station for simulating temperature and humidity at conditions labeled caution, extreme caution, and danger; (e) wearable biosensors measuring PPG, EDA, and S.T. signals.

\subsection{Chamber room}

The study was performed in an environmental chamber room with three different climatic conditions to simulate exposure to heat stress at the three levels of caution, extreme caution, and danger. The climatic conditions were simulated by controlling the temperature and humidity level. Such categorization was performed using the Humidex scale. This index calculates the temperature and humidity required to induce various levels of heat stress exposure. In this vein, for a condition of caution regarding heat stress, the temperature and humidity were set to $84.2 \mathrm{~F}^{\circ}$ and $40 \%$. Correspondingly, for extreme caution, temperature and humidity were set to $87.8 \mathrm{~F}^{\circ}$ and $45 \%$. Likewise, to simulate the condition of danger, the temperature and humidity were set to $89.6 \mathrm{~F}^{\circ}$ and $55 \%$, respectively.

\subsection{Data collection procedure}

All the subjects were asked to wear a non-intrusive wristband sensor (i.e., Empatica E4 wristband). They were instructed to perform roofing and materials handling tasks in the environmental chamber room. The roofing task involved nine minutes of hammering nails into a roofing surface angled $30^{\circ}$ from horizontal, as well as attaching and adjusting shingles. The materials handling task required subjects for six minutes to move concrete blocks from a wheelbarrow, place it on the roofing surface, and put it back in the wheelbarrow. Each subject was asked to perform the tasks in the chamber room for 15 minutes. Between the tests, they rested for 10 minutes. During each task, S.T., EDA, and PPG biosignals were captured at a sampling rate of $4 \mathrm{~Hz}, 64 \mathrm{~Hz}$, and $4 \mathrm{~Hz}$, respectively. The sensor promptly transmitted the data to a portable device. By using the sensor's application programming interface (API), the device was able to upload the biosignals onto an online server in near real-time. 


\section{Data analysis}

\subsection{Noise removal}

Interpretation of the signals can be affected by several kinds of noises. These come from changes in the body (i.e., excessive movement, etc.) and extrinsic changes (i.e., electrodes popping, environmental noises, ambient light, thermal noises, etc.) [41-44]. Robust noise removal decontaminated the biosignals of the major artifacts. Such an approach smooths the way for precise inferences of metrics based on the physiological signals. To better eliminate the adverse effects of signal noises, this study selected noise removal techniques based on the sources and modality of capture.

The PPG sensor determines the changes in blood flow by calculating the differences between the wavelengths of the original lights and the one reflected from the subject's tissue [45]. Environmental factors such as ambient light, thermal noise, and electromagnetic sources are more likely to induce high-frequency artifacts [45]. To remove them, a frequency-based filtering technique (i.e., bandpass filter) within the range of $0.5-4 \mathrm{~Hz}$ was applied. An EDA sensor records the signals by passing a minuscule amount of current between the electrodes in contact with the skin [41]. Hence, the measurement is vulnerable to different types of noises, namely electrode artifacts, excessive movement, and adjustment of sensors due to disruptions in the skin-electrode interface [41-44]. A high pass filter with a cut off frequency of $0.05 \mathrm{~Hz}$ was applied to the EDA signals. Furthermore, a rolling filter was used to remove large magnitude noises caused by excessive movement and electrode pressure. Different filtering methods (e.g., a Hampel filter and a finite impulse response filter) were applied to remove artifacts from S.T. signals. To remove outliers, a Hampel filter was used. A finite impulse response filter was applied to smooth the S.T. signals and to avoid aliasing in the data.

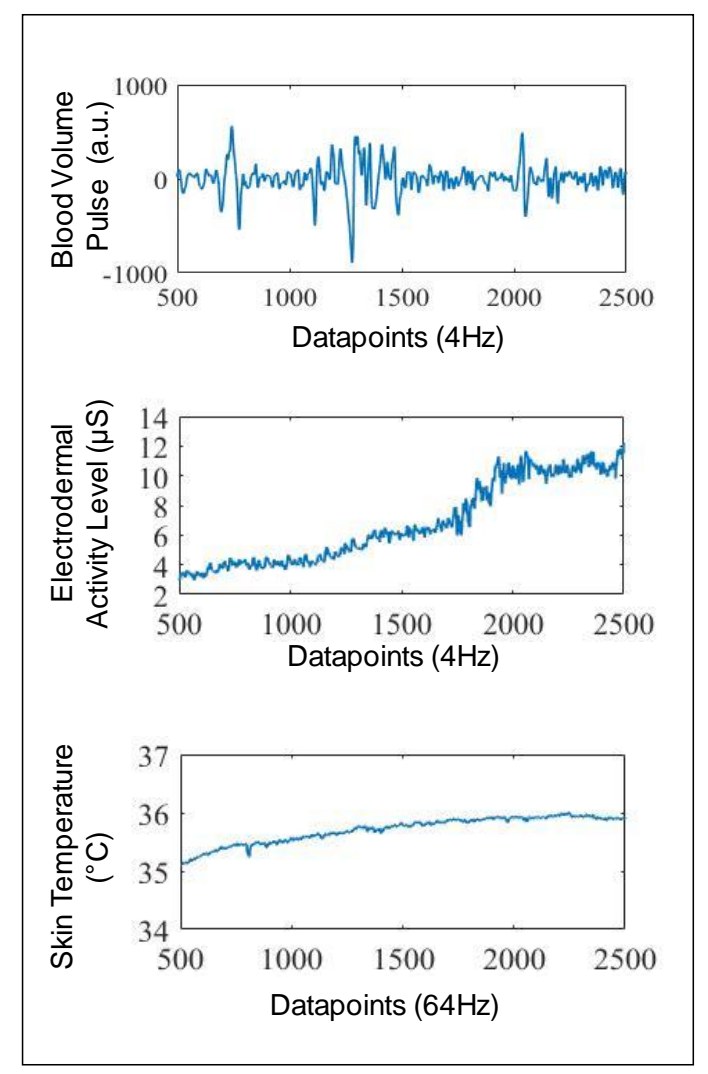

A

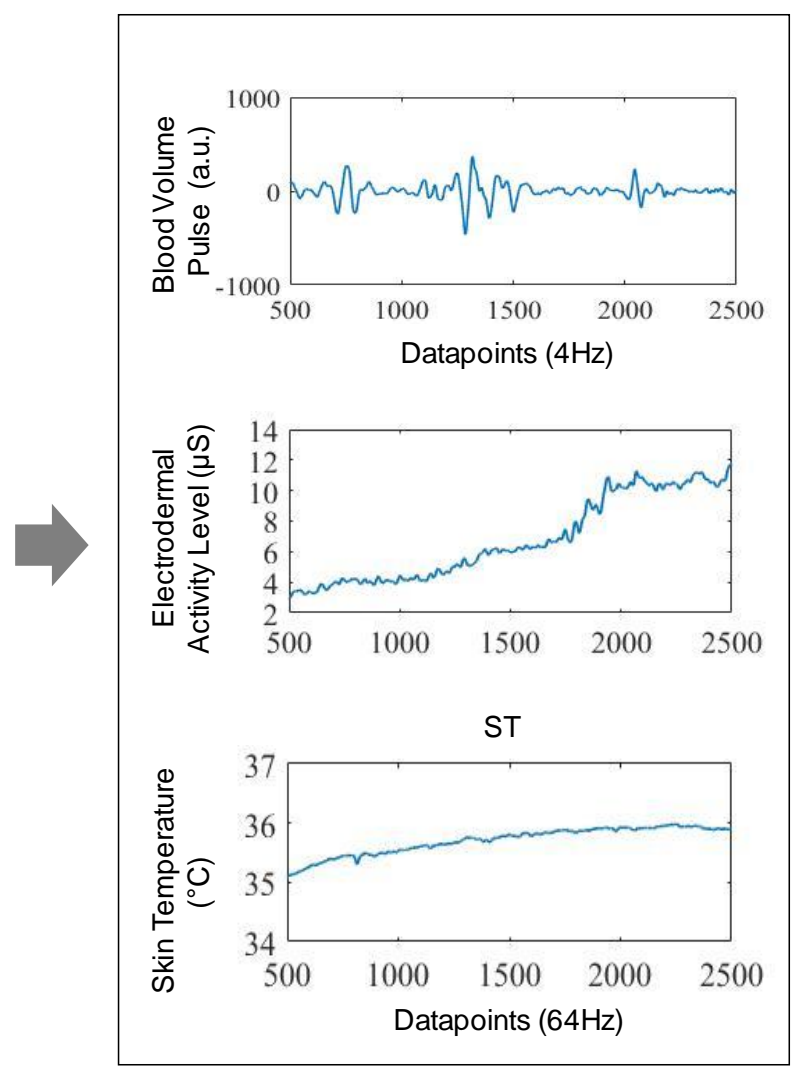

B

Fig. 2. PPG, EDA, and S.T. signals noise removal: (A) Raw signals; (B) Filtered signals

\subsection{Physiological metrics}

Several metrics were calculated after removing signal noise. From the PPG signals, three metrics were extracted, heart rate (H.R.), mean frequency, and median frequency. H.R. was determined from the processed PPG signal by counting the number of peaks in the alternating current of the signals. Also, the 
median value and mean frequency were extracted in the frequency domain to examine the changes in the subjects' cardiac activity.

Similarly, several metrics were extracted from EDA signals. These results included mean value, median value, and variance. EDL and EDR were extracted from the EDA signals to more achieve more precise results. EDL has been regarded as a suitable measure of sympathetic activity induced by long term stress $[46,47]$. On the other hand, EDR reflects short term responses to external stimuli $[48,49]$. In this regard, a deconvolution approach was applied for decomposing EDA signals into continuous signals of tonic and phasic activity to measure EDL and EDR, respectively. The metrics from S.T. in time and frequency domain included mean skin temperature (MST), mean frequency, and median frequency. These values were calculated after applying a finite impulse response filter to monitor skin temperature changes working under different exposures to heat stress.

\subsection{Statistical analysis}

To analyze the statistical differences among levels of heat stress exposure, the Wilcoxon signed-rank test was performed on the metrics. Specifically, this non-parametric comparison was used to determine the statistical differences across climatic conditions because the data did not satisfy the normal distribution assumption (Shapiro-Wilk test [50]). This lack was probably due to the low sample size [51]. In this study, the statistical comparison tests were performed with a 0.05 level of significance.

\section{Results}

Fig. 3 represents the distribution of the calculated metrics pertinent to the PPG, H.R., median frequency, and mean frequency, for all the subjects across all heat stress exposure. There were no statistically significant differences among the metrics computed for low, medium, and high climatic conditions.
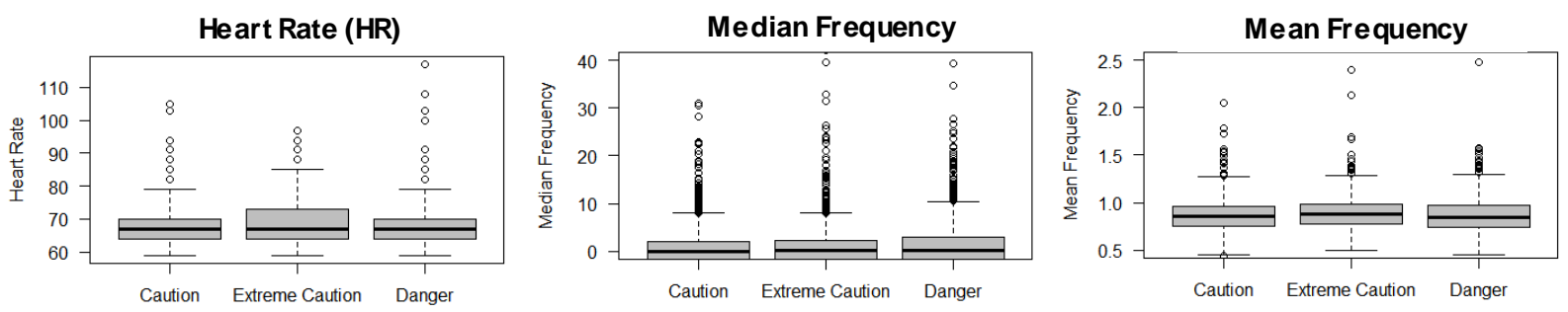

Fig. 3. Metrics values derived from PPG.

On the other hand, there were significant differences $(P$-value $<0.05)$ between the mean value, median value, and variance of EDA in the time domain. As seen in Fig. 4, as the heat stress level increases, these metrics exhibit a significant increase. More specifically, the Wilcoxon signed test indicated that the mean value for the EDA signals is significantly higher under medium heat stress than low heat stress ( $z=-5.05$, $p<0.05)$. Likewise, the test revealed a significant difference in the mean value of EDA signals between medium and high levels of heat stress $(z=-6.56, p<0.05)$. The test also corroborated a significant difference in the median value $(z=-5.05, p<0.05)$ and variance value $(z=-6.69, p<0.05)$ of the EDA signals across low and medium heat stress conditions. Similarly, the test showed that these metrics were significantly higher in high heat stress conditions than under medium heat stress $(z=-6.53, p<0.05$ for the median value and $z=$ $-4.39, p<0.05$ for the variance value). As for the EDR and EDL values, a similar trend can be observed among levels of exposure to heat stress (Fig. 4). The Wilcoxon signed test substantiated that the EDR values at medium heat stress are statistically higher than the values at low heat stress $(z=-5.0649, p<0.05)$. The results of the non-parametric test demonstrated that EDR changes from medium to high heat stress $(z=-$ 4.136, $p<0.05$ ). Similarly, EDL values exhibit a significant increase as the condition changed from low to medium heat stress $(z=-4.91, p<0.05)$, and from the medium to high heat stress $(z=-6.5277, p<0.05)$. 

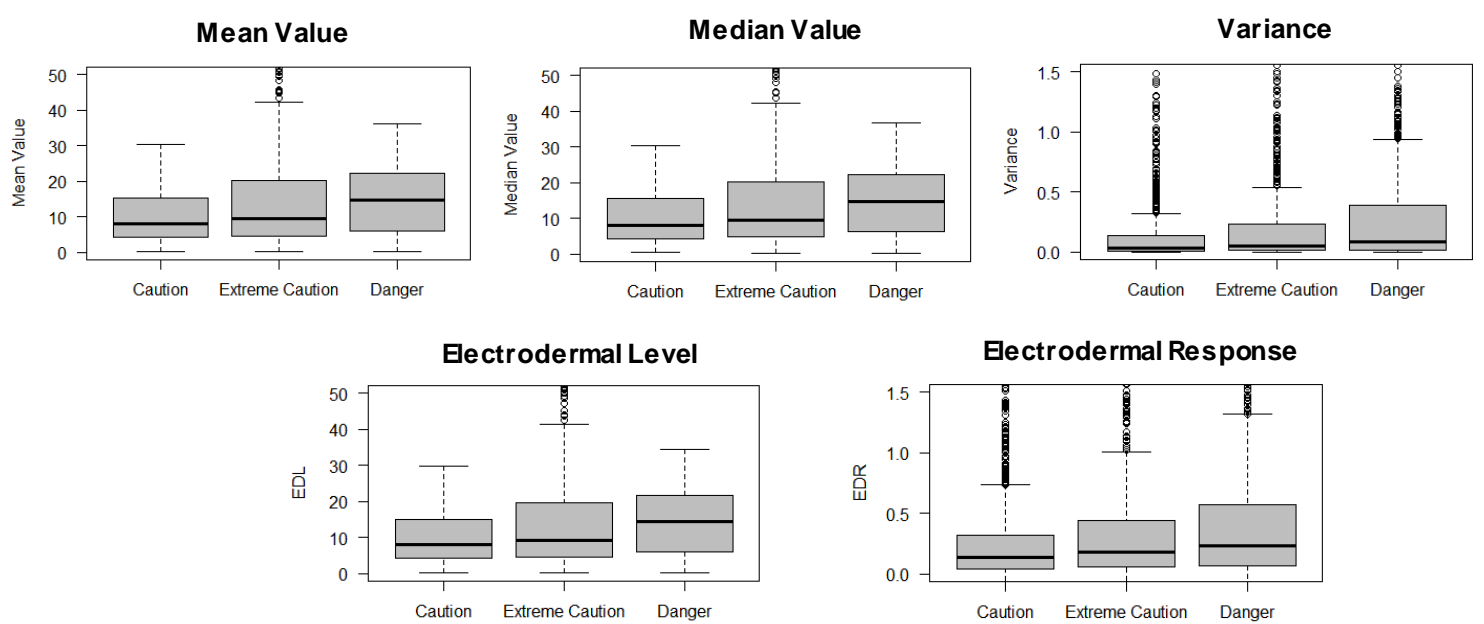

Fig. 4. Metrics values derived from EDA.

Meanwhile, differences in MST extracted from S.T. signals were captured while subjects were working under different levels of heat exposure. As seen in Fig. 5, the MST value is higher in the conditions with high heat stress. The Wilcoxon signed-rank test indicated that the MST value is significantly higher under medium heat stress conditions than in low heat $(z=-12.9, p<0.05)$ Also, the test stated that MST values significantly increased from medium to high heat stress conditions $(z=-21.3, p<0.05)$. On the other hand, metrics extracted from S.T. in the frequency domain (mean frequency and median frequency) failed to show any clear difference across levels of heat stress.
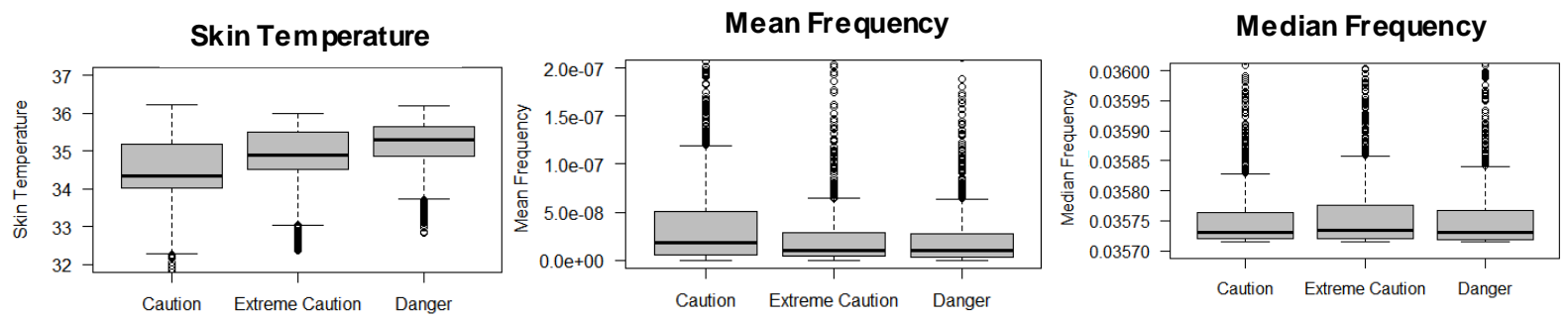

Fig. 5. Metrics values extracted from S.T. (MST, mean frequency, and median frequency)

\section{Conclusion}

This study was designed to investigate the capability of using physiological signals collected from a wristband sensor to capture the responses of the body due to exposure to heat stress. Metrics from PPG, EDA, and S.T. signals were collected from 10 healthy subjects performing two construction tasks under different environmental conditions. The results showed there is a significant difference in the metrics of EDA and S.T. among different levels of heat stress exposure. While the subjects performed the two tasks, roofing and materials handling, in thermally high stress conditions, the values of metrics from the EDA signals in the time domain (mean value, median value, and variance value) generally increased. The results indicated that EDL and EDR values increased with elevation in heat stress. Also, the findings revealed that MST extracted from S.T. signals exhibited significant differences under different climatic conditions. This pattern of the increase can be an appropriate criterion for the selection of relevant biosignals and the development of interpretive models for heat stress prediction and assessment. Such an attempt also augments our understanding of workers' physiological mechanisms under acute environmental conditions. As a critical step toward safer workplaces, this deeper understanding of human physiology can lend itself well to the design and development of wearable sensor-based health monitoring systems for a near realtime and objective assessment of worker heat stress in the field, which provides good opportunities to improve construction safety management. In addition, this study provides a promising avenue for personalized heat stress monitoring from wearable sensors by exploring the impact of heat stress exposure on key physiological responses. Future investigations can be more accurately performed through additional experiments with more significant numbers of subjects, physical activities, and naturalistic working environments. 
Proceedings of the Creative Construction e-Conference (2020) 004

Available online at e-2020.creative-construction-conference.com/proceedings/

\section{References}

[1] T.S. Abdelhamid, J.G. Everett, Physiological demands during construction work, J. Constr. Eng. Manag. 128 (2002) $427-437$. https://doi.org/10.1061/(ASCE)0733-9364(2002)128:5(427)

[2] J.K. Yates, Productivity improvement for construction and engineering: Implementing programs that save money and time, American Society of Civil Engineers (ASCE), 2014. https://doi.org/10.1061/9780784413463.

[3] U. Gatti, S. Schneider, G.M.-A. in Construction, undefined 2014, Physiological condition monitoring of construction workers, Elsevier. (n.d.). https://doi.org/10.1016/j.autcon.2014.04.013

[4] J. Xiang, P. Bi, D. Pisaniello, A. Hansen, Health Impacts of Workplace Heat Exposure: An Epidemiological Review, Ind. Health. 52 (2014) 91-101. https://doi.org/10.2486/indhealth.2012-0145.

[5] T. Kjellstrom, S. Gabrysch, B. Lemke, K. Dear, The "hothaps" programme for assessing climate change impacts on occupational health and productivity: An invitation to carry out field studies, Glob. Health Action. 2 (2009). https://doi.org/10.3402/gha.v2i0.2082.

[6] E.G. Hanna, T. Kjellstrom, C. Bennett, K. Dear, Climate change and rising heat: Population health implications for working people in Australia, Asia-Pacific J. Public Heal. 23 (2011) 14S - 26. https://doi.org/10.1177/1010539510391457.

[7] B. Tawatsupa, L.L.Y. Lim, T. Kjellstrom, S.A. Seubsman, A. Sleigh, J. Chokhanapitak, C. Churewong, S. Hounthasarn, S. Khamman D. Pandee, S. Pangsap, T. Prapamontol, J. Puengson, Y. Sangrattanakul, S.A. Seubsman, B. Somboonsook, N. Sripaiboonkij, P. Somsamai, D. Vilainerun, W. Wimonwattanaphan, C. Bain, E. Banks, C. Banwell, B. Caldwell, G. Carmichael, T. Dellora, J. Dixon, S. Friel, D. Harley, M. Kelly, L. Lim, Roderick McClure, Anthony McMichael, T. Mark, A. Sleigh, L. Strazdins, V. Yiengprugsawan, Association between occupational heat stress and kidney disease among 37816 workers in the thai cohort study (TCS), J. Epidemiol. 22 (2012) 251-260. https://doi.org/10.2188/jea.JE20110082.

[8] B. Tawatsupa, L.-Y. Lim, T. Kjellstrom, S. Seubsman, A. Sleigh, The association between overall health, psychological distress, and occupational heat stress among a large national cohort of 40,913 Thai workers, Glob. Health Action. 3 (2010) 5034. https://doi.org/10.3402/gha.v3i0.5034.

[9] D.M. Gubernot, G.B. Anderson, K.L. Hunting, Characterizing occupational heat-related mortality in the United States, 2000-2010: An analysis using the census of fatal occupational injuries database, Am. J. Ind. Med. 58 (2015) 203-211. https://doi.org/10.1002/ajim.22381.

[10] D. Bonauto, R. Anderson, E. Rauser, B. Burke, Occupational heat illness in Washington State, 1995-2005, Am. J. Ind. Med. 50 (2007) 940-950. https://doi.org/10.1002/ajim.20517.

[11] G. Hoorfarasat, M. Jafari, L. Omidi, S. Salehpour, S. Khodakarim, N. Haydarnezhad, Correlation between Heat Strain Score Index and WBGT Index with Physiological Parameters in a Glass Manufacturing Plant, (2015).

[12] V. Venugopal, J. Chinnadurai, R. Lucas, T. Kjellstrom, Occupational Heat Stress Profiles in Selected Workplaces in India, Int. J. Environ. Res. Public Health. 13 (2015) 89. https://doi.org/10.3390/ijerph13010089.

[13] M.Y. Beshir, J.D. Ramsey, Heat stress indices: A review paper, Int. J. Ind. Ergon. 3 (1988) 89-102. https://doi.org/10.1016/01698141(88)90012-1.

[14] J. Zhao, S. Lorenzo, N. An, W. Feng, ... L.L.-J. of E., undefined 2013, Effects of heat and different humidity levels on aerobic and anaerobic exercise performance in athletes, Elsevier. (n.d.). https://doi.org/10.1016/j.jesf.2013.04.002

[15] X. Shi, N. Zhu, G.Z.-B. and environment, undefined 2013, The combined effect of temperature, relative humidity and work intensity on human strain in hot and humid environments, Elsevier. (n.d.). https://doi.org/10.1016/j.buildenv.2013.07.016.

[16] S. Hwang, S.H. Lee, Wristband-type wearable health devices to measure construction workers' physical demands, Autom. Constr. 83 (2017) 330-340. https://doi.org/10.1016/j.autcon.2017.06.003.

[17] H. Jebelli, B. Choi, S. Lee, Application of Wearable Biosensors to Construction Sites. II: Assessing Workers' Physical Demand, J. Constr. Eng. Manag. 145 (2019) 04019080. https://doi.org/10.1061/(ASCE)CO.1943-7862.0001710

[18] H. Jebelli, S. Hwang, S.H. Lee, EEG-based workers' stress recognition at construction sites, Autom. Constr. 93 (2018) 315-324. https://doi.org/10.1016/j.autcon.2018.05.027.

[19] M. Habibnezhad, J. Puckett, H. Jebelli, A. Karji, M.S. Fardhosseini, S. Asadi, Neurophysiological testing for assessing construction workers' task performance at virtual height, Autom. Constr. 113 (2020) 103143. https://doi.org/10.1016/j.autcon.2020.103143.

[20] B. Choi, H. Jebelli, S.H. Lee, Feasibility analysis of electrodermal activity (EDA) acquired from wearable sensors to assess construction workers' perceived risk, Saf. Sci. 115 (2019) 110-120. https://doi.org/10.1016/j.ssci.2019.01.022.

[21] H. Jebelli, M.M. Khalili, S. Lee, Mobile EEG-Based Workers' Stress Recognition by Applying Deep Neural Network, in: Adv. Informatics Comput. Civ. Constr. Eng., Springer International Publishing, 2019: pp. 173-180. https://doi.org/10.1007/978-3-03000220-6_21.

[22] H. Jebelli, B. Choi, S. Lee, Application of Wearable Biosensors to Construction Sites. I: Assessing Workers' Stress, J. Constr. Eng. Manag. 145 (2019) 04019079. https://doi.org/10.1061/(ASCE)CO.1943-7862.0001729

[23] C.R. Ahn, S.H. Lee, C. Sun, H. Jebelli, K. Yang, B. Choi, Wearable Sensing Technology Applications in Construction Safety and Health, J. Constr. Eng. Manag. 145 (2019). https://doi.org/10.1061/(ASCE)CO.1943-7862.0001708

[24] T. Kjellstrom, I. Holmer, B. Lemke, Workplace heat stress, health and productivity-an increasing challenge for low and middle-income countries during climate change, Glob. Health Action. 2 (2009). https://doi.org/10.3402/gha.v2i0.2047.

[25] R.R. Pryor, B.L. Bennett, F.G. O'connor, J.M.J. Young, C.A. Asplund, H. Sciences, M. Bethesda, D. Bennett, O.' Connor, Medical Evaluation for Exposure Extremes: Heat, 2015. https://doi.org/10.1016/j.wem.2015.09.009.

[26] K. Lundgren, K. Kuklane, C. Gao, I. Holmér, Effects of heat stress on working populations when facing climate change, Ind. Health. 51 (2013) 3-15. https://doi.org/10.2486/indhealth.2012-0089.

[27] Niosh, Criteria for a Recommended Standard: Occupational Exposure to Heat and Hot Environments, n.d.

[28] F.B. Nerbass, R. Pecoits-Filho, W.F. Clark, J.M. Sontrop, C.W. McIntyre, L. Moist, Occupational Heat Stress and Kidney Health: From Farms to Factories, Kidney Int. Reports. 2 (2017) 998-1008. https://doi.org/10.1016/j.ekir.2017.08.012.

[29] M. Elgendi, R. Fletcher, I. Norton, M. Brearley, D. Abbott, N.H. Lovell, D. Schuurmans, On time domain analysis of photoplethysmogram signals for monitoring heat stress, Sensors (Switzerland). 15 (2015) 24716-24734. https://doi.org/10.3390/s151024716.

[30] J.S. Cuddy, M. Buller, W.S. Hailes, B.C. Ruby, Skin temperature and heart rate can be used to estimate physiological strain during exercise in the heat in a cohort of fit and unfit males., Mil. Med. 178 (2013) e841-7. https://doi.org/10.7205/MILMED-D-12-00524.

[31] W. Boucsein, D.C. Fowles, S. Grimnes, G. Ben-Shakhar, W.T. Roth, M.E. Dawson, D.L. Filion, Publication recommendations for electrodermal measurements SOCIETY FOR PSYCHOPHYSIOLOGICAL RESEARCH AD HOC COMMITTEE ON ELECTRODERMAL MEAS-URES, (2012). https://doi.org/10.1111/j.1469-8986.2012.01384.x.

[32] J. Allen, Photoplethysmography and its application in clinical physiological measurement, Physiol. Meas. 28 (2007). https://doi.org/10.1088/0967-3334/28/3/R01. 
Proceedings of the Creative Construction e-Conference (2020) 004

Available online at e-2020.creative-construction-conference.com/proceedings/

[33] M.J. Buller, W.A. Latzka, M. Yokota, W.J. Tharion, D.S. Moran, A real-time heat strain risk classifier using heart rate and skin temperature., Physiol. Meas. 29 (2008) N79-85. https://doi.org/10.1088/0967-3334/29/12/N01.

[34] J. Achten, A.E. Jeukendrup, Heart rate monitoring: Applications and limitations, Sport. Med. 33 (2003) 517-538. https://doi.org/10.2165/00007256-200333070-00004.

[35] M. Benedek, C. Kaernbach, A continuous measure of phasic electrodermal activity, J. Neurosci. Methods. 190 (2010) 80-91. https://doi.org/10.1016/j.jneumeth.2010.04.028.

[36] M. Poh, ... N.S.-I. transactions on, undefined 2010, A wearable sensor for unobtrusive, long-term assessment of electrodermal activity, leeexplore.leee.Org. (n.d.). https://doi.org/10.1109/TBME.2009.2038487

[37] W.I. Cranston, Thermoregulation and the Skin, in: 1989: pp. 213-221. https://doi.org/10.1007/978-3-642-73797-8_12.

[38] A. Aryal, A. Ghahramani, B. Becerik-Gerber, Monitoring fatigue in construction workers using physiological measurements, Autom. Constr. 82 (2017) 154-165. https://doi.org/10.1016/j.autcon.2017.03.003.

[39] J.-H. Choi, V. Loftness, Investigation of human body skin temperatures as a bio-signal to indicate overall thermal sensations, (2012). https://doi.org/10.1016/j.buildenv.2012.07.003.

[40] A. Hunter, C.A. Gibson, Z. Mbambo, M. Lambert, T. Noakes, The effects of heat stress on neuromuscular activity during endurance exercise, Pflugers Arch. Eur. J. Physiol. 444 (2002) 738-743. https://doi.org/10.1007/s00424-002-0841-x.

[41] W. Boucsein, D.C. Fowles, S. Grimnes, G. Ben-Shakhar, W.T. Roth, M.E. Dawson, D.L. Filion, Publication recommendations for electrodermal measurements, Psychophysiology. 49 (2012) 1017-1034. https://doi.org/10.1111/j.1469-8986.2012.01384.x.

[42] K.T. Sweeney, T.E. Ward, S.F. McLoone, Artifact removal in physiological signals-practices and possibilities, IEEE Trans. Inf. Technol. Biomed. 16 (2012) 488-500. https://doi.org/10.1109/TITB.2012.2188536.

[43] S. Taylor, N. Jaques, W. Chen, S. Fedor, A. Sano, R. Picard, Automatic identification of artifacts in electrodermal activity data, in: Proc. Annu. Int. Conf. IEEE Eng. Med. Biol. Soc. EMBS, Institute of Electrical and Electronics Engineers Inc., 2015: pp. $1934-1937$. https://doi.org/10.1109/EMBC.2015.7318762.

[44] G. Lee, B. Choi, H. Jebelli, C.R. Ahn, S. Lee, Reference Signal-Based Method to Remove Respiration Noise in Electrodermal Activity (EDA) Collected from the Field, in: American Society of Civil Engineers (ASCE), 2019: pp. 17-25. https://doi.org/10.1061/9780784482438.003.

[45] H. Jebelli, B. Choi, H. Kim, S. Lee, Feasibility study of a wristband-type wearable sensor to understand construction workers' physical and mental status, in: Constr. Res. Congr. 2018 Constr. Inf. Technol. - Sel. Pap. from Constr. Res. Congr. 2018, American Society of Civil Engineers (ASCE), 2018: pp. 367-377. https://doi.org/10.1061/9780784481264.036.

[46] J.J. Braithwaite, D. Derrick, G. Watson, R. Jones, M. Rowe, A Guide for Analysing Electrodermal Activity (EDA) \& Skin Conductance Responses (SCRs) for Psychological Experiments, n.d.

[47] C. Kappeler-Setz, F. Gravenhorst, J. Schumm, B. Arnrich, G. Tröster, Towards long term monitoring of electrodermal activity in daily life, Pers. Ubiquitous Comput. 17 (2013) 261-271. https://doi.org/10.1007/s00779-011-0463-4.

[48] A. Greco, G. Valenza, ... A.L.-I.T. on, undefined 2015, cvxEDA: A convex optimization approach to electrodermal activity processing, leeexplore.leee.Org. (n.d.). https://doi.org/10.1109/TBME.2015.2474131

[49] M. Benedek, C.K.-J. of neuroscience methods, undefined 2010, A continuous measure of phasic electrodermal activity, Elsevier. (n.d.). https://www.sciencedirect.com/science/article/pii/S0165027010002335 (accessed March 26, 2020).

[50] P. Mishra, C.M. Pandey, U. Singh, A. Gupta, C. Sahu, A. Keshri, Descriptive statistics and normality tests for statistical data, Ann. Card. Anaesth. 22 (2019) 67-72. https://doi.org/10.4103/aca.ACA_157_18.

[51] J. Rochon, M. Gondan, M. Kieser, To test or not to test: Preliminary assessment of normality when comparing two independent samples, BMC Med. Res. Methodol. 12 (2012) 81. https://doi.org/10.1186/1471-2288-12-81. 